\title{
Influência do nível socioeconômico sobre comportamentos de risco para transtornos alimentares em adolescentes
}

\author{
Socioeconomic influence on eating disorders risk behaviors in adolescents \\ Karin Louise Lenz Dunker1', Cássia Peres Bonar Fernandes', Daniel Carreira Filho²
}

\section{RESUMO}

Objetivo: Investigar comportamentos de risco para transtornos alimentares (TA) em adolescentes de diferentes níveis socioeconômicos. Método: 183 adolescentes do sexo feminino de 15 a 18 anos de duas escolas (pública e particular) foram avaliadas quanto à renda e escolaridade dos pais, estado nutricional, comportamentos de risco para TA (Teste de Atitudes Alimentares - EAT-26), atitudes socioculturais quanto à aparência (Escala das Atitudes Socioculturais Voltadas para Aparência - SATAQ-3), percepção corporal (escala de silhuetas) e uso de dietas. Resultados: A média do escore do EAT-26, assim como a porcentagem de adolescentes consideradas de risco foram semelhantes entre as escolas $(p>0,05)$. No escore do SATAQ-3, verificou-se na escola particular um valor maior que da pública $(p<0,05)$. 0 número de adolescentes que superestimavam o peso foi semelhante nas escolas $(p>0,05)$, e as que apresentaram sobrepeso/obesidade tiveram escores maiores no EAT-26. Metade das adolescentes das escolas já fez dieta, sendo que a frequência do uso de dietas esteve associada com o maior escore do EAT-26. Conclusões: Comportamentos de risco foram encontrados em grande parte da população estudada, mas esses não estiveram associados com o nível socioeconômico.

\section{ABSTRACT}

Objective: To investigate eating disorders (ED) risk behaviors at adolescents from different socioeconomic status. Method: 183 girls 15-18 years old from two schools (public and private) were evaluated about parent's education and income, nutritional status, ED risk behavior (Eating Attitudes Test - EAT-26), sociocultural attitudes toward appearance (SATAQ-3), corporal perception (silhouettes scale) and dieting. Results: Mean score of EAT-26, as well as percentage of risky girls were similar at both schools ( $p>0.05)$. At SATAQ-3 score, it was noticed a higher value at private school compared to public one $(p<0.05)$. Girls that overestimate weight were similar at

\section{Keywords}

Eating disorders, socioeconomic status, adolescents. both schools ( $p>0.05$ ), and girls who were overweight/obese had a higher score at EAT-26. Half of the girls from both schools have already diet, and dieting frequency was associated with a higher score at EAT-26. Conclusions: Risky behaviors were found at most of the studied population, however these were not associated with socioeconomic status.

1 Universidade Cruzeiro do Sul, SP.

2 Centro Universitário Unimódulo, Caraguatatuba, SP. 


\section{INTRODUÇÃO}

A adolescência é um período de transformações biológicas e psicossociais, em que alterações do corpo ocorrem paralelamente à constituição da personalidade, refletindo assim no desenvolvimento afetivo-emocional. A mudança do corpo e peso transforma a imagem mental que o adolescente faz de si, podendo levá-lo a sentir-se estranho, desproporcional, com uma imagem corporal negativa'

A diferença entre o peso atual (em função das alterações da puberdade) e o ideal promovido pela mídia, associada com a necessidade de adaptação junto à sociedade e uma baixa autoestima, são fatores que determinam uma insatisfação corporal. Essa pode levar muitas vezes ao início de comportamentos de risco para transtornos alimentares (TA), como o uso de dietas restritivas e de métodos de controle de peso (vômitos autoinduzidos, laxantes, diuréticos, atividade física excessiva) para emagrecer'.

A prevalência dos TA, como anorexia nervosa e bulimia nervosa, varia de 0,5 a 1,0\% em países como os Estados Uni$\operatorname{dos}^{18}$. No Brasil, não há dados publicados de prevalência, mas observa-se um aumento evidente no número de casos em ambulatórios voltados ao atendimento dessas doenças como o Ambulatório de Bulimia e Transtornos Alimentares (AMBULIM) do Hospital das Clínicas, considerado o maior da América Latina, e também a publicação de trabalhos nacionais demonstrando a insatisfação corporal e o uso de dietas em adolescentes $2,8,11,23,28$.

Os fatores etiológicos dos TA são baseados na vulnerabilidade biológica, predisposição psicológica, e por algum tempo foi discutida na literatura a influência da classe social.

Afirma-se que há uma apresentação maior de casos de TA nas classes média e alta. Os estudos realizados nessa área são poucos, e os resultados são controversos, pois alguns afirmam que os comportamentos estão mais associados a indivíduos das classes média e alta, outros os associam com a classe baixa, e ainda outros dizem que essa associação não é totalmente correta, havendo pouco ou nenhum efeito da classe social. Os autores concluem que essa relação ainda precisa ser mais estudada $2,3,5,10,16,19,21,22,24$.

No Brasil não existem estudos que relacionem os comportamentos de TA e a satisfação corporal em adolescentes de acordo com o nível socioeconômico. Os poucos estudos descritivos de comportamento alimentar e de satisfação corporal do adolescente brasileiro têm levado os pesquisadores a empreender estudos epidemiológicos sobre as atitudes alimentares dos jovens. O intuito desses estudos é facilitar o reconhecimento precoce das populações de risco de desenvolver TA, proporcionando a prevenção das formas graves e resultando num melhor prognóstico 2,11.

Por se tratar de um país onde ainda existem muitas desigualdades sociais, e que está passando por um processo de transição nutricional, no qual a desnutrição vem diminuindo e problemas como obesidade e transtornos alimentares vêm aumentando, faz-se necessário realizar um estudo de investigação em populações de diferentes situações socioeconômicas, observando as diferenças quanto à presença de comportamentos de TA, percepção corporal, atitudes socioculturais quanto à aparência e uso de dietas para emagrecer.

\section{MÉTODO}

Trata-se de um estudo de corte transversal realizado com adolescentes estudantes de escolas públicas e particulares localizadas na região leste do município de São Paulo - SP. Do total de 10 escolas públicas e 14 particulares contatadas, somente 2 particulares e 2 públicas autorizaram a pesquisa. Funcionários das escolas passaram em sala de aula convidando as alunas a participarem da pesquisa, portanto a adesão foi voluntária. Consideraram-se como critérios de inclusão: apresentar interesse voluntário em participar da pesquisa, ser do gênero feminino, ter entre 15 e 18 anos completos e ter permissão dos pais para participar do estudo.

Os dados de escolaridade e renda familiar em salários mínimos (SM) foram obtidos das adolescentes por meio de preenchimento de questionário pelos pais ou responsáveis. Os dados não permitiram uma definição da classe social. As comparações de situação socioeconômica foram feitas baseadas no maior/menor nível de escolaridade dos membros da família e de renda familiar.

O estado nutricional foi determinado a partir das medidas de peso e altura - índice de massa corporal (IMC), o qual foi classificado em baixo peso, eutrofia, e sobrepeso/ obesidade, de acordo com os percentis da Organização Mundial da Saúde ${ }^{31}$. Foram verificados o peso e a estatura por meio de balança digital e fita métrica inelástica afixada à parede.

A identificação dos indivíduos com comportamentos de TA realizou-se a partir do questionário Teste de Atitudes Alimentares (EAT-26), validado em adolescentes do sexo feminino de 12 a 18 anos $^{6}$. É um teste psicométrico muito utilizado em estudos epidemiológicos para rastrear indivíduos supostamente suscetíveis ao desenvolvimento de TA, e indica a presença de comportamentos de risco, como restrição alimentar e preocupação com peso. Consideraram-se como adolescentes de risco aquelas que apresentaram um escore maior ou igual a 20 no questionário.

A Escala das Atitudes Socioculturais Voltadas para Aparência (SATAQ-3) ${ }^{27}$ foi utilizada para avaliar o grau em que a mídia pode influenciar o indivíduo em sua imagem corporal e nos comportamentos de risco para TA. A versão em português, com tradução e retrotradução, foi feita com autorização prévia do autor do questionário, por Dunker ${ }^{12}$. 
É um questionário que apresenta uma validade convergente com questionários que medem imagem corporal e TA, e os escores são mais altos em indivíduos com TA e de risco para TA.

A escala de silhuetas de Stunkard ${ }^{26}$, que consiste de 9 figuras esquemáticas do corpo magro ao corpo obeso, foi utilizada para avaliar a percepção corporal das adolescentes. Para verificar se as adolescentes superestimavam o peso, os números das silhuetas foram definidos para adolescentes em valores de percentis de IMC e idade ${ }^{20,31}$, considerando-se: 2 (P15), 3 (P32,5), 4 (P50), 5 (P85), $\geq 6$ (P95).

Por fim, as adolescentes foram questionadas quanto ao uso de dietas para perda de peso e sua frequência.

Para a análise dos dados, realizou-se uma análise descritiva da média e desvio-padrão para avaliar a variabilidade do escore do EAT-26 segundo estado nutricional, uso de dietas, renda e escolaridade dos pais, e o teste "t-student" para verificar diferenças do escore SATAQ-3 e do EAT-26 entre as escolas.

O teste de qui-quadrado $\left(X^{2}\right)$ de Pearson foi utilizado com o objetivo de explorar as possíveis associações entre o tipo de escola e as variáveis socioeconômicas, estado nutricional e percepção corporal.

Em todos os testes, fixou-se um $\alpha=5 \%(p \leq 0,05)$ para o nível de rejeição da hipótese de nulidade, assinalando-se com um asterisco $(*)$ valores significativos.

Os dados foram tabulados e analisados pelo software estatístico Statistical Package for Social Science (SPSS), versão 10.0 for Windows.

A pesquisa foi aprovada pelo Comitê de Ética em Pesquisa da Universidade Cruzeiro do Sul, e todos os indivíduos assinaram termo de consentimento após esclarecimento sobre o estudo.

\section{RESULTADOS}

Participaram do estudo 183 adolescentes, sendo 116 alunas de escola pública e 67 de escola particular, com idades entre 15 e 18 anos.

Em relação à situação socioeconômica, observou-se que a maioria dos chefes de família da escola pública tem ensino médio completo/incompleto $(43,9 \%)$ e uma renda de até 5 salários mínimos (77,9\%), enquanto na escola particular, a maioria $(53,6 \%)$ tem ensino superior completo/incompleto e uma renda maior que 5 a 15 salários mínimos (61,2\%), sendo que houve associação estatisticamente significante entre essas variáveis e o tipo de escola (Tabela 1).

A maioria das adolescentes da escola pública $(68,1 \%)$ e da particular (73,13\%) estava eutrófica, enquanto 14,7\% das adolescentes da escola pública e 10,4\% da particular apresentavam sobrepeso/obesidade (Tabela 2).
Tabela 1. Renda e escolaridade dos pais das adolescentes. São Paulo-2007.

\begin{tabular}{|c|c|c|c|c|c|}
\hline & \multicolumn{2}{|c|}{ Pública } & \multicolumn{2}{|c|}{ Particular } & \multirow[t]{2}{*}{ p-valor* } \\
\hline & $n$ & $\%$ & n & $\%$ & \\
\hline \multicolumn{6}{|l|}{ Escolaridade do chefe da família } \\
\hline Analfabeto & 7 & 6,0 & 2 & 2,9 & \multirow{6}{*}{$<0,05$} \\
\hline Ensino Fundamental C/I & 21 & 18,1 & 7 & 10,4 & \\
\hline Ensino Médio C/I & 51 & 43,9 & 16 & 23,8 & \\
\hline Curso Superior C/I & 30 & 25,8 & 36 & 53,6 & \\
\hline Pós-graduação & 7 & 6,0 & 6 & 8,9 & \\
\hline Total & 116 & 100,0 & 67 & 100,0 & \\
\hline \multicolumn{6}{|l|}{ Renda familiar } \\
\hline Até 5 SM & 90 & 77,9 & 12 & 17,9 & \multirow{4}{*}{$<0,05$} \\
\hline 5 a $15 \mathrm{SM}$ & 25 & 21,5 & 41 & 61,2 & \\
\hline Acima de 15 SM & 1 & 0,9 & 14 & 20,9 & \\
\hline Total & 116 & 100,0 & 67 & 100,0 & \\
\hline
\end{tabular}

Tabela 2. Distribuição percentual das alunas segundo estado nutricional, escore do EAT-26, uso de dietas e insatisfação corporal. São Paulo - 2007.

\begin{tabular}{|c|c|c|c|c|c|}
\hline & \multicolumn{2}{|c|}{ Pública } & \multicolumn{2}{|c|}{ Particular } & \multirow[t]{2}{*}{ p-valor* } \\
\hline & $\mathrm{n}$ & $\%$ & $\mathrm{n}$ & $\%$ & \\
\hline \multicolumn{6}{|l|}{ Estado nutricional } \\
\hline Baixo peso & 20 & 17,2 & 11 & 16,5 & $>0,05$ \\
\hline Eutrofia & 79 & 68,1 & 49 & 73,1 & \\
\hline Sobrepeso/obesidade & 17 & 14,7 & 7 & 10,4 & \\
\hline \multicolumn{6}{|l|}{ Escore do EAT-26 } \\
\hline$\geq$ de 20 & 33 & 28,4 & 23 & 34,3 & $>0,05$ \\
\hline$<$ de 20 & 83 & 71,6 & 44 & 65,7 & \\
\hline \multicolumn{6}{|l|}{ Uso de dietas } \\
\hline Não fazem & 58 & 50,0 & 34 & 50,7 & \\
\hline 1 a 2 vezes & 25 & 21,5 & 13 & 21,4 & $>0,05$ \\
\hline 3 a 4 vezes & 13 & 11,2 & 12 & 16,0 & \\
\hline 5 vezes ou + & 20 & 17,2 & 8 & 11,9 & \\
\hline Superestimam o peso & 25 & 21,5 & 18 & 26,8 & \\
\hline Baixo peso & 9 & 36 & 6 & 33,3 & \\
\hline Eutrofia & 15 & 60 & 11 & 61,2 & $>0,05$ \\
\hline Sobrepeso/obesidade & 1 & 4 & 1 & 5,5 & \\
\hline Total & 25 & 100 & 18 & 100 & \\
\hline
\end{tabular}

A média do escore do EAT-26 nas duas escolas foi considerada abaixo do risco (<20) (Tabela 3). O escore da escola particular foi um pouco acima da pública, assim como a porcentagem de alunas com escore acima de 20 (34,3\%), comparada com a escola pública $(28,4 \%)$ (Tabela 2$)$, no entanto essa diferença não foi estatisticamente significante.

$\mathrm{Na}$ avaliação do escore do EAT-26, segundo as variáveis socioeconômicas, estado nutricional e uso de dietas, algumas diferenças entre as escolas foram encontradas (Tabela 3). Na variável escolaridade e renda, ocorreu uma diferença significativa de escore entre as escolas dos pais com ensino médio e com renda de 5 a 15 SM, indicando que os pais da escola particular apresentam maiores escores que o da pública. 
Tabela 3. Média e desvio-padrão do escore do EAT-26 segundo as variáveis socioeconômicas, estado nutricional e uso de dietas das adolescentes e escore do SATAQ-3. São Paulo - 2007.

\begin{tabular}{|c|c|c|c|c|c|}
\hline & \multicolumn{2}{|c|}{$\begin{array}{l}\text { Pública } \\
\text { Média (DP) }\end{array}$} & \multicolumn{2}{|c|}{$\begin{array}{l}\text { Particular } \\
\text { Média (DP) }\end{array}$} & p-valor* \\
\hline \multicolumn{6}{|l|}{ Escolaridade do chefe da familia } \\
\hline Analfabeto & 14 & 7,9 & 16 & 0,7 & 0,770 \\
\hline Ensino fundamental C/I & 20 & 13,2 & 18 & 6,5 & 0,773 \\
\hline Ensino Médio C/I & 16 & 9 & 21 & 13 & $0,015^{*}$ \\
\hline Curso Superior C/I & 13 & 8,9 & 19 & 13,2 & 0,071 \\
\hline Pós-graduação & 17 & 9,8 & 17 & 5,6 & 0,869 \\
\hline \multicolumn{6}{|l|}{ Renda familiar } \\
\hline Até 5 SM & 16 & 10,7 & 14 & 10,6 & 0,535 \\
\hline 5 a 15 SM & 14 & 6,9 & 20 & 11,7 & $0,034^{*}$ \\
\hline Acima de 15 SM & 6 & - & 20 & 12,6 & - \\
\hline \multicolumn{6}{|l|}{ Estado Nutricional } \\
\hline Baixo peso & 9 & 6,4 & 15 & 8,1 & $0,038^{*}$ \\
\hline Eutrofia & 16 & 9,1 & 19 & 12,6 & 0,085 \\
\hline Sobrepeso/obesidade & 23 & 11,8 & 23 & 11 & 0,961 \\
\hline \multicolumn{6}{|l|}{ Uso de dietas } \\
\hline Não fazem & 10 & 6,2 & 14 & 6,6 & $0,008^{*}$ \\
\hline 1 a 2 vezes & 19 & 2,2 & 16 & 4,9 & 0,219 \\
\hline 3 a 4 vezes & 21 & 6,6 & 29 & 12,4 & $0,031^{*}$ \\
\hline 5 vezes ou + & 25 & 10,9 & 30 & 18,7 & 0,349 \\
\hline Escore total EAT-26 & 16 & 9,9 & 19 & 11,8 & 0,058 \\
\hline Escore SATAQ-3 & 86 & 15,0 & 90 & 16,0 & $0,043^{*}$ \\
\hline
\end{tabular}

$C / I=$ completo/incompleto; ${ }^{*}$ Teste t-student; ${ }^{* * S M}=$ salários mínimos $(15 M=R \$ 350,00)$.

Em relação ao estado nutricional (Tabela 3), observa-se que há diferença entre as escolas em relação às adolescentes com baixo peso, sendo que os valores do escore do EAT-26 foram maiores na escola particular, mas não considerados de risco; enquanto as adolescentes com sobrepeso/obesidade das duas escolas tiveram um escore maior que 20 no EAT-26, sendo consideradas, portanto, um grupo de risco.

No escore total do SATAQ-3, verificou-se que a escola particular teve um valor médio maior e estatisticamente significante comparado com o da escola pública (Tabela 3).

$\mathrm{Na}$ comparação dos valores de IMC atual com os relacionados à silhueta, nota-se que o número de adolescentes que superestimam o peso foi semelhante entre as escolas, ou seja, 21,5\% na escola pública e 26,8\% na particular. A maioria dessas estava eutrófica, seguida das com baixo peso. No entanto, o estado nutricional não esteve associado com o tipo de escola, como pode ser observado na Tabela 2.

$\mathrm{Na}$ Tabela 2 observa-se que cerca de metade das adolescentes já fez dieta; na Tabela 3 verifica-se um comportamento semelhante nas escolas: quanto maior o número de vezes de realização de dietas, maior o escore do EAT-26; e também que o escore das meninas que não fazem dieta e das que já fizeram de 3 a 4 dietas é maior na escola particular, diferença considerada estatisticamente significante.

\section{DISCUSSÃO}

O presente estudo encontrou diferenças significativas nas variáveis socioeconômicas entre as escolas. A maioria dos pais das adolescentes da escola pública tende a ter uma escolaridade e renda menor que os pais da escola particular, o que indica uma diferença de classe social.

Alguns estudos nacionais vêm relacionando a classe social com o estado nutricional, segundo os quais a prevalência de sobrepeso é maior em indivíduos com melhor condição socioeconômica e consequentemente maior acesso aos alimentos, como foi observado neste estudo ${ }^{13,29}$.

No entanto, observa-se em outros estudos em escolas públicas e/ou particulares uma variabilidade de prevalência, o que demonstra que a situação socioeconômica não está necessariamente relacionada ao estado nutricional 2,11,15,25.

Considerando as diferenças de escore do EAT-26 entre as escolas, observou-se na escola particular um escore maior, assim como um maior número de meninas com valores acima de 20 no escore. Apesar das diferenças significativas observadas na Tabela 3 entre os pais da escola pública e da particular, a escolaridade e renda não podem ser determinantes de maior risco, pois os escores maiores observados na particular em função da escolaridade não foram observados na pública.

Alguns trabalhos recentes da literatura internacional, com adolescentes na faixa etária de 12 a 18 anos, apresentaram resultados semelhantes ao presente estudo, ou seja, a situação socioeconômica não teve relação com a presença de comportamentos de transtornos alimentares 3,5,21,24.

Outros estudos encontraram relação do nível socioeconômico com os sintomas de TA. Costa et al. ${ }^{10}$ verificaram em adolescentes portugueses com um maior nível socioeconômico um maior risco para desenvolver TA.

Um estudo realizado com uma grande amostra de crianças e adolescentes alemães, com idades entre 11 e 17 anos, observou que os adolescentes de baixo nível socioeconômico apresentam quase duas vezes mais sintomas de TA do que aqueles pertencentes às classes socioeconômicas mais altas ${ }^{19}$. Power et al. ${ }^{22}$, em seu estudo com adolescentes equatorianas, verificaram que um baixo nível socioeconômico esteve relacionado com escores do EAT maiores.

Por fim o estudo de Alves et al. ${ }^{2}$, realizado com uma grande amostra de adolescentes de Florianópolis de escolas públicas e particulares, encontrou resultados controversos. O risco de TA não esteve associado à renda, no entanto encontrou-se uma associação entre rede pública de ensino e a presença de sintomas de TA.

O presente estudo encontrou mais de 30\% das adolescentes com escores superiores a 20 no EAT-26, ou seja, com risco de desenvolver TA. Segundo, Benavente et al. ${ }^{4}$, prevalências de sintomas em torno de $9 \%$ são consideradas abaixo da média, indicando uma característica positiva da população estudada em relação aos TA. Por outro lado, prevalências maiores de $20 \%$ são bastante preocupantes. 
Diversos estudos encontraram uma taxa de EAT de $6 \%$ a $32 \%$ entre adolescentes do gênero feminino 2,11,17,25,28.

Em relação aos resultados encontrados no SATAQ-3, pode-se observar que houve diferença entre as escolas, e que os valores encontrados neste estudo são semelhantes aos encontrados no estudo de Dunker ${ }^{12}$, em que se aplicou o questionário em adolescentes de 12 a 14 anos de uma escola particular, com média de escore do EAT-26 semelhante.

Segundo Thompson et al..27, o questionário avalia o grau em que o indivíduo internaliza os conceitos de magreza ideal, ou seja, a extensão com que o indivíduo cognitivamente aceita as normas da sociedade de padrão de beleza, ao ponto de modificar seu comportamento para se aproximar desses padrões. A internalização da magreza, o ideal de beleza e a pressão pela magreza (por parte da família, amigos, namorados e mídia) predizem um aumento significante a curto prazo na insatisfação corporal em adolescentes.

$\mathrm{Na}$ avaliação da percepção do peso atual, verificou-se nas duas escolas uma porcentagem de alunas com baixo peso ou adequado que superestimavam o peso. A percepção de um peso acima do normal em adolescentes com peso adequado é um comportamento típico desse estágio de vida $8,11,12,23,28$.

Por outro lado um maior escore no EAT-26 esteve relacionado com um maior IMC, ou seja, a indivíduos com sobrepeso/obesidade, assim como ocorreu em outros estudos que relacionaram a pontuação no EAT-26 com o estado nutricional, indicando que os adolescentes com obesidade têm maior número de atitudes alimentares anormais do que aqueles eutróficos ou de baixo peso, podendo esse fato predispô-los ao início de um TA 7,11.

Os resultados do estudo demonstram que metade das adolescentes das escolas já fizeram dietas, com pequenas diferenças significativas de acordo com o tipo de escola, sendo mais comum na escola particular. O hábito de fazer dieta é um comportamento comum, principalmente em meninas, devido à maior preocupação dessas com o peso, como descrito em estudos nacionais 13,23,28.

Sabe-se que os TA, invariavelmente, têm início com uma perda de peso resultante de doença física ou dieta para emagrecer em razão de excesso de peso real ou imaginário. O comportamento de "fazer dieta" está associado a sintomas mentais e físicos como fadiga, ansiedade, depressão, constipação, amenorreia, raciocínio lento, baixo desempenho na escola e decréscimo do crescimento; e ainda com comportamentos alimentares como pular refeições, comer de forma compulsiva e ter baixo consumo de cálcio e ferro, nutrientes de particular importância na adolescência ${ }^{1,12}$.

A restrição calórica, com objetivo da perda de peso, também está associada com a obsessão pela comida; ansiedade com alimentação; interferência na regulação natural da fome; episódios do comer compulsivo; o hábito de ocultar, esconder e evitar alimentos; e desnutrição, e ainda com alguns sinais agudos como irritabilidade e retração social ${ }^{1,12}$.

Os comportamentos como a preocupação com peso e forma corporal, e restrição alimentar são muito frequentes entre as adolescentes, independentemente do estado nutricional e dos parâmetros socioeconômicos, ocorrendo, portanto, em todas as classes sociais, sendo mais prevalentes nas sociedades industrializadas 3,5,21,24.

Supostamente, a associação com classes econômicas mais privilegiadas refletiria um viés dos primeiros estudos epidemiológicos baseados em registros hospitalares, cuja população atendida pertencia de fato a essas classes $^{18}$. Segundo Favoro et al.14, o grau de urbanização de uma região pode influenciar no desenvolvimento dos TA sem necessariamente haver associação com o nível socioeconômico da população. Todavia, questiona-se também se as regiões menos urbanizadas e industrializadas conseguem conter a influência da globalização na divulgação dos ideais de beleza relacionados à forma do corpo.

Alguns estudos reforçam a teoria de que a mídia tem uma grande influência sobre os adolescentes, pois ela gera uma pressão pelo emagrecimento a qualquer custo, podendo aumentar o risco de esses desenvolverem um $T A^{9,30}$.

\section{CONCLUSÃO}

Os resultados encontrados demonstram que comportamentos de risco para TA, como a preocupação com peso e restrição alimentar, não têm associação com a situação socioeconômica, mas apresentam associação com sobrepeso/obesidade e a maior frequência de uso de dietas.

\section{REFERÊNCIAS}

1. Alvarenga M, Dunker KLL. Padrão e comportamento alimentar na anorexia e na bulimia nervosa. In: Philippi ST, Alvarenga M. Transtornos alimentares: uma visão nutricional. São Paulo: Manole, 2004. p.131-48

2. Alves E, Vasconcelos FAG, Calvo MCM, Neves J. Prevalência de sintomas de anorexia nervosa e insatisfação com a imagem corporal em adolescentes do sexo feminino do município de Florianópolis, Santa Catarina, Brasil. Cad Saúde Pública. 2008;24:503-12.

3 Bauer M, Kirchengast S. Body composition, weight status, body image and weight control practices among female adolescents from eastern Austria. Antropol Anz. 2006;64:321.

4. Benavente MD, Morilla FR, Leal CM, Benjumea MVH. Factores de riesgo relacionados con trastornos en la conducta alimentaria en una comunidad de escolares. Aten Primaria. 2003:32:403-9.

5. Bergström H, Elfhag K. Socioeconomic differences in obese patients with and without eating disorders. Eat Weight Disord. 2007;12:e98-103.

6. Biguetti F, Santos CB, Santos JE, Ribeiro RPP. Tradução e validação do eating attitudes test em adolescentes do sexo feminino de Ribeirão Preto, São Paulo. J Bras Psiquiatria. 2004;53:339-46

7. Borresen R, Rosenvinge JH. Body dissatisfaction and dieting in 4,952 Norwegian children aged 11-15 years: less evidence for gender and age differences. Eat Weight Disord. 2003:8:238-41. 
8. Branco LM, Hilário MOE, Cintra IP. Perception and satisfaction with body image in adolescents and correlations with nutrition status. Rev Psiq Clín. 2006;33:292-6.

9. Chow J. Adolescents' perceptions of popular teen magazines. J Adv Nurs. 2004;48:132-9.

10. Costa C, Ramos E, Severo M, Barros H, Lopes C. Determinants of eating disorders symptomatology in Portuguese adolescents. Arch Pediatr Adolesc Med. 2008;162:1126-32.

11. Dunker KLL, Philippi ST. Avaliação do estado nutricional e percepção corpórea de adolescentes com sintomas de anorexia nervosa. Pediatria Moderna. 2003;39:12-16.

12. Dunker KLL. Programa de prevenção de comportamentos de risco para transtornos alimentares em adolescentes: estudo piloto. São Paulo; 2006. [Tese de Doutorado - FCF/FEA/ FSP/USP].

13. Dutra CL, Araújo CL, Bertoldi AD. Prevalência de sobrepeso em adolescentes: um estudo de base populacional em uma cidade no Sul do Brasil. Cad Saúde Pública. 2006;22:151-62.

14. Favoro A, Ferrara S, Santonastaso P. The spectrum of eating disorders in young women: a prevalence study in a general population sample. Psychosom Med. 2003;65:701-8.

15. Ferreira JES, Veiga GV. Confiabilidade (teste-reteste) de um questionário simplificado para triagem de adolescentes com comportamentos de risco para transtornos alimentares em estudos epidemiológicos. Rev Bras Epidemiol. 2008;11:393-401.

16. Gard MCE, Freeman CP. The dismantling of a myth: a review of eating disorders and socieconomic status. Int J Eat Disord. 1996;20:1-12.

17. González-Juárez C, Pérez-Pérez E, Martín CB, Mitja PI, Roy PR, Vázquez TEP. Detección de adolescentes en riesgo de presentar trastornos de la alimentación. Aten Primaria. 2007;39:189-94.

18. Hay PJ. Epidemiologia dos transtornos alimentares: estado atual e desenvolvimentos futuros. Rev Bras Psiquiatr. 2002;24:13-7.

19. Holling H, Schlack R. Eating disorders in children and adolescents, first results of the German Health Interview and Examination Survey for Children and Adolescents (KiGGS). Bundesgesundheitsblatt Gesundheitsforschung Gesundheitsschutz. 2007;50:794-9.

20. Must A, Phillips SM, Stunkard AJ, Naumova EN. Expert opinion on body mass index percentiles for figure drawings at menarche. Int J 0besity. 2002;26:876-9.
21. Preti A, Pinna C, Nocco S, Pilia S, Mulliri E, Micheli V, et al. Rural/urban differences in the distribution of eating disorder symptoms among adolescents from community samples. Aust N Z J Psychiatry. 2007;41:525-35.

22. Power Y, Power L, Canadas MB. Low socioeconomic status predicts abnormal eating attitudes in Latin American female adolescents. Eat Disord. 2008;16:136-45.

23. Reato LFN, Harada RM, Hatakeyama TT, Kitaura AR, Nagaoka BM, Perestrelo VB. Hábitos alimentares, comportamentos de risco e prevenção de transtornos alimentares em adolescentes do Ensino Médio. Rev Paul Pediatr. 2007;25:22-6.

24. Soh NL, Touyz S, Dobbins TA, Surgenor LJ, Clarke S, Kohn MR, et al. Restraint and eating concern in North European and East Asian women with and without eating disorders in Australia and Singapore. Aust N Z J Psychiatry. 2007;41:536-45.

25. Souza-Kaneshima AM, França AA, Kneube DPF, Kaneshima EM. Ocorrência de anorexia nervosa e distúrbio de imagem corporal em estudantes do ensino médio de uma escola da rede pública da cidade de Maringá, Estado do Paraná. Acta Sci Health Sci. 2006;28:119-27.

26. Stunkard A. Old and new scales for the assessment of body image. Perceptual and Motor Skills. 2000;90:930

27. Thompson JK, Berg PVD, Roehrig M, Guarda AS, Heinber LJ. The sociocultural attitudes towards appearance sclae-3 (SATAQ-3): development and validation. Int J Eat Disord; 2004; 35:293-304.

28. Vilela JEM, Lamounier JA, Dellaretti Filho MA, Barros Neto JR, Horta GM. Transtornos alimentares em escolares. J Pediatr (Rio J). 2004;80:49-54.

29. Wang Y, Monteiro C, Popkin BM. Trends of obesity and underweight in older children and adolescents in the United States, Brazil, China, and Russia. Am J Clin Nutr. 2002;75:971-7.

30. Wiseman CV, Sunday SR, Becker AE. Impact of the media on adolescent body image. Child Adolesc Psychiatr Clin N Am. 2005;14:453-71.

31. World Health Organization. The use and interpretation of anthropometry physical status. Geneve: Report of a WHO study group; 1995. (Technical Report Series 854). 